Case of tel angi ect at i c/i nf I ammat ory hepat ocel I ul ar adenoma ari sing in a pati ent with pri mary sclerosing chol angi ti s

\begin{tabular}{|l|l|}
\hline 著者 & $\begin{array}{l}\text { Nayl ee Hsu, Har ada Keni chi, I gar ashi Saya, } \\
\text { Tohda Gen, Yamantot o Nakot o, Ren Xi ang Shan, } \\
\text { Osawa Takeshi, Hasegawa Yasuhi ro, Takahashi } \\
\text { Nor i o, Nakanuma Yasuni }\end{array}$ \\
\hline $\begin{array}{l}\text { j our nal or } \\
\text { publ i cat i on ti t l e }\end{array}$ & Hepat ol ogy Resear ch \\
\hline vol une & 42 \\
\hline nunber & 6 \\
\hline page r ange & $611-618$ \\
\hline year & $2012-06-01$ \\
\hline URL & ht t p: //hdl . handl e. net /2297/31404 \\
\hline
\end{tabular}




\section{A case of telangiectatic/inflammatory hepatocellular adenoma arising in a patient with primary sclerosing cholangitis}

Hsu Maylee ${ }^{1)}$, Kenichi Harada ${ }^{1)}$, Saya Igarashi ${ }^{1)}$, Gen Tohda ${ }^{2)}$, Makoto Yamamoto ${ }^{2)}$, Xiang shan Ren ${ }^{1)}$,

Takeshi Osawa $^{3)}$, Yasuhiro Hasegawa ${ }^{3)}$, Norio Takahashi ${ }^{4)}$, and Yasuni Nakanuma ${ }^{1)}$

Human Pathology, Kanazawa University Graduate School of Medicine, Kanazawa, Japan ${ }^{1)}$. Internal medicine $^{2)}$, Surgery ${ }^{3)}$, Radiology ${ }^{4)}$, Fukui Kosei Hospital, Fukui, Japan.

Short running title; Hepatocellular adenoma in PSC

Key Words: hepatocellular adenoma, primary sclerosing cholangitis, ulcerative colitis

\section{Address correspondence to:}

Yasuni Nakanuma, M.D.

Department of Human Pathology

Kanazawa University Graduate School of Medicine

Kanazawa 920-8640, Japan

FAX : (0)76-234-4229 (Japan)

TEL : (0)76-265-2195 (Japan)

E-mail : pbcpsc@kenroku.kanazawa-u.ac.jp 


\begin{abstract}
Hepatocellular adenomas (HCAs) have recently identified as a heterogeneous group, differing based on genotypic as well as morphologic characteristics. HCAs are most frequently found in females on oral contraception. A type of HCA, inflammatory HCA is also known as telangiectatic HCA and previously referred to as telangiectatic focal nodular hyperplasia. We present the first case of HCA arising from the liver with primary sclerosing cholangitis (PSC). This case is a 30'-year-old man with a past medical history of PSC, ulcerative colitis, and diabetes mellitus. A routine ultrasonography for PSC detected the gradually enlarged intrahepatic mass. Liver biopsy could reveal the diagnosis of telangiectatic/inflammatory HCA by morphological and immunohistochemical analyses. Partial hepatectomy was performed and the resected liver was pathologically diagnosed as the telangiectatic/inflammatory HCA arising in PSC. This is the first case report of such an association and here we review the current developments and literature of this rare tumor and the association with an activated inflammatory related tumorgenetic pathway and PSC.
\end{abstract}




\section{INTRODUCTION}

With recent molecular advances profiling and subclassifying hepatocellular adenomas (HCAs), the entity of telangiectatic hepatocellular adenoma, previously thought to be a subtype of focal nodular hyperplasia $(\mathrm{FNH})$, has now been reclassified as a variant of the inflammatory HCA subtype. ${ }^{1-4}$ Telangiectatic/inflammatory HCA is a rare benign tumor characterized as a welldemarcated, unencapsulated tumor with partial lobulation, portal tract-like structures with dystrophic or thickened arteries surrounded by an inflammatory infiltrate and/or ductular reaction, and scattered areas of peliosis and sinusoidal dilation. ${ }^{1,5,6}$ The diagnosis is based on these key morphologic features and can be further supported with immunohistochemistry and molecular genotyping. ${ }^{1-7}$

HCAs are most frequently and classically found in females on oral contraception. ${ }^{8}$ Additionally, telangiectatic/inflammatory HCA have specifically been linked to an increased body mass index and a generalized systemic inflammatory condition. ${ }^{6}$ Other rare, but well-documented, associations with HCAs include: glycogensosis, ${ }^{4,9}$ familial adenomatous polyposis coli, ${ }^{4,10,11}$ Danazol treatment, ${ }^{4}, 12,13$ portal canal shunt, ${ }^{4}$ Cushing syndrome, ${ }^{4}$ and diabetes mellitus with liver adenomatosis. ${ }^{14}$ Here we report the first case of a telangiectatic/inflammatory HCA occurring in a male patient with primary sclerosing cholangitis (PSC).

\section{CASE REPORT}

Clinical course: This case is a $30^{\prime}$-year-old man at diagnosis of HCA. The patient first presented with acute hepatitis at the age of 11 and was then diagnosed with PSC at the age of 16 . When he was 15 years old he presented with hematochezia and diarrhea and was then diagnosed with ulcerative colitis (UC). He was also diagnosed with diabetes mellitus (DM) type 2, and at age 28 he was noted to have cholelithiasis. Since then, his UC has been in remission and his DM has been wellcontrolled with medication. He has no history of steroid use and is a social drinker.

He initially presented with a low echo lesion in the left lobe found during a routine PSC ultrasonography screening. The lesion was followed and gradually enlarged in size. Two years later, the lesion was biopsied and then the patient underwent a partial hepatectomy of the left lobe. On physical examination on admission, he has a body mass index (BMI) of $31(92 \mathrm{~kg}$ and $172 \mathrm{~cm})$, and a blood pressure of $106 / 76 \mathrm{mmHg}$. Laboratory data are as follows (underline denotes abnormal data) : WBC 5200/ml, RBC $476 \times 10^{4} / \mathrm{ml}$, PLT $26.8 \times 10^{4} / \mathrm{ml}$, ESR $24 \mathrm{~mm} / \mathrm{h}$, C-reactive protein (CRP) 0.5 mg/dl, TP 7.4 g/dl, Alb 3.9 g/dl, T-Bil 0.8 mg/dl, ALP 706 IU/1, ChE 5051 IU/1, AST 43 IU/1, ALT 91 IU/1, LDH 178 IU/1, y-GTP 718 IU/l, AMY 31 IU/l, ZTT 19.2 U, LDL-C 166 mg/dl, TG 182 mg/dl, HDL-C 52 mg/dl, UN 10 mg/dl, S-Cr 0.56 mg/dl, Na 138 mEq/l , K 4.3 mEq/l, Cl 101 mEq/l , Ca 9.3 mg/dl, UA 4.7 mg/dl, HbAlc 7.0 \%, IgG 1688 mg/dl, IgA 267 mg/dl, IgM 147 mg/dl, ANA (- , < 40), 
$\operatorname{AMA}(-,<20), \operatorname{M} 2 \mathrm{Ab}(-,<5), \operatorname{SSA}(-,<5), \operatorname{SSB}(-,<5)$, C-ANCA 222 U/1, HBs-Ag (-), HCV-Ab(-), AFP 3.4ng/ml, and PIVKA-II $39 \mathrm{mAU} / \mathrm{ml}$.

Imaging: The initial screening ultrasonography revealed a low echo lesion in the left lobe and the surrounding liver showed fatty change. CT scans were then performed to further evaluate the hepatic mass. An unenhanced CT scan showed irregular fatty change. On a dynamic contrast CT scan, the mass revealed enhancement in the hepatic arterial phase and persistent enhancement in the portalvenous and equilibrium phase (Fig.1). No other masses were found in the liver. After 10 months of follow up, ultrasonography and CT scan showed enlargement of the mass. On MRI imaging the mass showed high intensity on both T1 and T2 weighted images. The T2-weighted fat suppressed image and the out of phase image also showed high intensity, which indicated that there was no fat component within the lesion. The SPIO-enhanced MRI showed iso-intensity compared to the surrounding liver, and the Gd-EOB-MRI showed strong enhancement on the arterial phase and iso-intensity in comparison with the surrounding liver on portal-venous and equilibrium phase. On the hepatobiliary phase, the lesion showed hypo-intensity (Fig.1), but it seemed to have a slight uptake of Gd-EOB-DTPA. From these images, tumor capsule was unclear. Overall, these findings suggest the hypervascular lesion showing a gradually enlargement. Especially, as differential diagnoses, focal nodular hyperplasia, angiomyolipoma, HCA, and hepatocellular carcinoma were considered. As for the background liver, MRCP and ERCP showed multifocal intrahepatic bile duct strictures suggesting PSC.

Histology of liver needle biopsy (Fig.2): Specimen contained tumorous and background areas. Tumorous lesion composed of uniform population of pale eosinophilic tumor cells resembling hepatocytes. Many of the tumor cells had glycogenated nuclei and no nuclear atypia was seen. Pericellular frame of reticulin fiber was well-preserved. A few portal tract-like structures lacking bile ducts were found in this lesion and around them mild lymphocytic infiltration and copper-binding proteins positive for Orcein staining were scattered.

Pathology of partially resected liver (Fig.3): Gross examination of the liver excision specimen revealed a non-encapsulated, well-circumscribed $2.6 \mathrm{~cm}$ soft tan nodule. The center of the nodule had several small foci of hemorrhage but no central scar or areas of fibrosis were identified. Histology revealed a well-demarcated nodule composed of uniform hepatocyte-like cells without cellular atypia. Within the tumor mass there were abundant portal tract-like structures, each containing two to three arteries. The arteries varied in size and caliber, some thickened, dystrophic and/or ectatic. Surrounding these portal tract-like structures was a brisk lymphoplasmacytic infiltrate with rare foci of an associated ductular proliferation. Areas of macrovesicular steatosis were identified within the tumor, predominately surrounding the portal tract-like structures. Peliosis and areas with sinusoidal dilation and congestion were also present. The background liver showed findings consisted with early 
stage of primary sclerosing cholangitis with a lymphoplasmacytic portal tract infiltrate centered on the bile ducts with bile duct damage and some areas of concentric fibrosis surrounding bile ducts (onionskin like lesion).

Immunohistochemistry: Immunohistochemistry using needle and surgically resected liver specimens demonstrated that the tumor cells stained positive for serum amyloid A (SAA, granular cytoplasmic staining pattern) and strongly positive for CRP (Fig.3) and had no nuclear staining for $\beta$ catenin. CD34 highlighted the arteries within the portal tract-like structures and also showed a sinusoidal inflow staining pattern (Fig.3). Cytokeratins 7 and 19 highlighted a mild ductular proliferation surrounding rare portal tract-like structures and also bile ducts and a ductular reaction within the background liver. Overall, these histopathologic and immunohistochemical findings were diagnostic and classic for a telangiectatic/inflammatory HCA. ${ }^{3-5}$

\section{DISCUSSION}

Recent molecular studies have shown that HCAs are composed of a heterogeneous group of tumors, differing based on hepatocyte nuclear factor (HNF)- $1 \alpha$ mutations, $\beta$-catenin activation, and morphologic phenotypes. ${ }^{1-7,15}$ Although several nomenclatures of HCA subclassification, according to new WHO classification edited in 2010, HCAs can now be subclassified into four categories, 1) HNF1 $\alpha$-inactivated HCA (tumors with HNF1 $\alpha$ mutation with steatosis, lack of cytologic abnormalities, no inflammatory infiltrate, and negative liver fatty acid protein expression), 2) $\beta$ catenin-activated HCA (tumors with $\beta$-catenin mutation with frequent cytologic abnormalities and pseudo-glandular formation), 3) Inflammatory HCA (telangiectatic/inflammatory HCA without HNF $1 \alpha$ or $\beta$-catenin activation with cytological abnormalities, ductular reaction, inflammatory infiltrates, and frequent gp130 mutation), 4) Unclassified HCA (tumors without any mutation/activation and no inflammatory infiltrate). ${ }^{1-4}$ 15, 16 This morphologic and genotypic classification is important for prognostic and diagnostic purposes, since those tumors with $\beta$-catenin activation have been shown to have an increased risk to progress to hepatocellular carcinoma. ${ }^{2}, 17,18$ Furthermore, these studies have also clarified the status of telangiectatic HCA, previously referred to as telangiectatic focal nodular hyperplasia. ${ }^{19}$ Telangiectatic HCAs are not only monoclonal tumors, ${ }^{1,5}$ but also share many features of inflammatory HCA, both morphologically and genotypically. ${ }^{1-7}$ Both telangiectatic and inflammatory HCAs fit into the third group of HCAs (telangiectatic/inflammatory $\mathrm{HCA}$ ), with the lack of HNF1 $\alpha$ mutation or $\beta$-catenin activation and with an inflammatory infiltrate surrounding portal tract like-structures. A risk of malignant transformation in telangiectatic/inflammatory HCA without $\beta$-catenin mutation might be rather low. ${ }^{16}$ Moreover, Dokmak et al, ${ }^{20}$ reported that patients with HCAs greater than $5 \mathrm{~cm}$, telangiectatic or unclassified subtypes, and men have an increased risk of complication of hepatic resection. The present male case 
was unlikely malignant transformation and the risk of complication of resection was also feared. However, the mass of this patent was progressively enlarged from $2.0 \mathrm{~cm}$ to $2.8 \mathrm{~cm}$ over the course of 10 months and the potential malignant transformation entirely could not be denied. Therefore, this patient desired a surgical treatment as a treatment choice.

While all types of HAs are classically found in females and are strongly associated with the use of oral contraception, ${ }^{8}$ the telangiectatic/inflammatory HCA subtypes have been specifically linked to obesity, alcohol, and most importantly to an inflammatory syndrome. Moreover, patients with telangiectatic/inflammatory HCA have been shown to have increased blood levels of CRP and fibrinogen and following surgical removal of the tumors, these patients subsequently have reduced blood level of these inflammatory makers. ${ }^{6}$ In the present case of telangiectatic/inflammatory HCA, however, the level of serum CRP was not increased. Because CRP level is affected by the size of tumor, as a possible reason, the tumor size of this patient is still small, compared with that of typical HCA. Also telangiectatic/inflammatory HCA characteristically have an immunohistochemical pattern of SAA and CRP overexpression and these markers are overexpressed only within the tumor hepatocytes. ${ }^{3}$ Moreover, the investigation of MRI findings for each type of HCAs has already reported. ${ }^{15}$ Telangiectatic/inflammatory HCA subtype is characterized by the high intensity signal on T2-weighted sequences and our present case also shows the similar MRI findings. Finally, direct evidence for an aberrant activation of an inflammatory pathway in these tumors has been shown with the identification of interleukin (IL)-6ST mutations, encoding gp130 receptor, occurring in $60 \%$ of telangiectatic/inflammatory HCAs. ${ }^{21}$ This mutation leads to an activation of the IL-6/STAT-3 signaling pathway, which provides a mechanism for the inflammatory infiltrate found within the tumors, the increased levels of circulating inflammatory markers in these patients, and may serve as a critical link to an inflammatory related tumorgenetic pathway. ${ }^{22}$

PSC is a well-known chronic inflammatory condition targeting the intrahepatic and extrahepatic large bile ducts, leading to fibrotic strictures and eventually biliary cirrhosis. The risk for cholangiocarcinoma is up to $13 \%,{ }^{23}$ and hepatocellular carcinoma is a rare complication. ${ }^{24}$ In this case report, the patient had a long-standing history of PSC and ulcerative colitis. No other reports have associated HCA with PSC. A literature search only revealed one cases report of a patient with PSC and $\mathrm{FNH}^{24}$ Furthermore this is the first report of a telangiectatic/inflammatory HCA associated with a specific underlying chronic inflammatory condition. This case is important in that it identifies PSC as a possible associated risk factor for the development of telangiectatic/inflammatory HCA. The pathogenesis for the development of cholangiocarcinoma arising in patients with PSC and other chronic inflammatory disorders of the bile ducts has been linked to an activation of the IL-6 and STAT3 pathway. ${ }^{25-29}$ This common inflammatory pathway of IL-6/STAT3 has also been welldocumented in colitis associated colon cancer in patients with inflammatory bowel disease. ${ }^{22}$ Given that telangiectatic/inflammatory HCA also are found to have an activated IL-6/STAT3 signaling 
pathway, perhaps this patient's underlying chronic inflammatory diseases of PSC and/or UC, may have predisposed him to the development of a telangiectatic/inflammatory HCA.

In summary, here we report the first case of a telangiectatic/inflammatory HCA associated with PSC. Further studies are necessary to identify a possibly link or associated inflammatory mechanism between PSC and telangiectatic/inflammatory HCA. 


\section{REFEERCES}

1. Paradis V, Benzekri A, Dargere D, et al. Telangiectatic focal nodular hyperplasia: a variant of hepatocellular adenoma. Gastroenterology 2004;126(5):1323-9.

2. Zucman-Rossi J, Jeannot E, Nhieu JT, et al. Genotype-phenotype correlation in hepatocellular adenoma: new classification and relationship with HCC. Hepatology 2006;43(3):515-24.

3. Bioulac-Sage P, Rebouissou S, Thomas C, et al. Hepatocellular adenoma subtype classification using molecular markers and immunohistochemistry. Hepatology 2007;46(3):740-8.

4. Bioulac-Sage P, Laumonier H, Couchy G, et al. Hepatocellular adenoma management and phenotypic classification: the Bordeaux experience. Hepatology 2009;50(2):481-9.

5. Bioulac-Sage P, Rebouissou S, Sa Cunha A, et al. Clinical, morphologic, and molecular features defining so-called telangiectatic focal nodular hyperplasias of the liver. Gastroenterology 2005;128(5):1211-8.

6. Paradis V, Champault A, Ronot $\mathrm{M}$, et al. Telangiectatic adenoma: an entity associated with increased body mass index and inflammation. Hepatology 2007;46(1):140-6.

7. Ahmad I, Iyer A, Marginean CE, et al. Diagnostic use of cytokeratins, CD34, and neuronal cell adhesion molecule staining in focal nodular hyperplasia and hepatic adenoma. Hum Pathol 2009;40(5):726-34.

8. Edmondson HA, Henderson B, Benton B. Liver-cell adenomas associated with use of oral contraceptives. N Engl J Med 1976;294(9):470-2.

9. Kishnani PS, Chuang TP, Bali D, et al. Chromosomal and genetic alterations in human hepatocellular adenomas associated with type Ia glycogen storage disease. Hum Mol Genet 2009;18(24):4781-90.

10. Jeannot E, Wendum D, Paye F, et al. Hepatocellular adenoma displaying a HNF1alpha inactivation in a patient with familial adenomatous polyposis coli. J Hepatol 2006;45(6):883-6.

11. Okamura Y, Maeda A, Matsunaga K, et al. Hepatocellular adenoma in a male with familial adenomatous polyposis coli. J Hepatobiliary Pancreat Surg 2009;16(4):571-4.

12. Bork K, Schneiders V. Danazol-induced hepatocellular adenoma in patients with hereditary angio-oedema. J Hepatol 2002;36(5):707-9.

13. Fermand JP, Levy Y, Bouscary D, et al. Danazol-induced hepatocellular adenoma. Am J Med 1990;88(5):529-30.

14. Reznik Y, Dao T, Coutant R, et al. Hepatocyte nuclear factor-1 alpha gene inactivation: cosegregation between liver adenomatosis and diabetes phenotypes in two maturity-onset diabetes of the young (MODY)3 families. J Clin Endocrinol Metab 2004;89(3):1476-80.

15. Ronot M, Bahrami S, Calderaro J, et al. Hepatocellular adenomas: accuracy of magnetic resonance imaging and liver biopsy in subtype classification. Hepatology 2011;53(4):1182-91. 
16. Bioulac-Sage P, Balabaud C, Wanless IR. Focal nodular hyperplasia and hepatocellular adenoma. In: Bosman FT, Carneiro F, Hruban RH, Theise ND, editors. WHO classification of tumors of the digestive system; World Health Organization of Tumors. 4th ed. Lyon: IARC; 2010. p. 198-204

17. Chen YW, Jeng YM, Yeh SH, et al. P53 gene and Wnt signaling in benign neoplasms: betacatenin mutations in hepatic adenoma but not in focal nodular hyperplasia. Hepatology 2002;36(4 Pt 1):927-35.

18. Ogawa K, Yamada Y, Kishibe K, et al. Beta-catenin mutations are frequent in hepatocellular carcinomas but absent in adenomas induced by diethylnitrosamine in $\mathrm{B} 6 \mathrm{C} 3 \mathrm{~F} 1$ mice. Cancer Res 1999;59(8):1830-3.

19. Wanless IR, Albrecht S, Bilbao J, et al. Multiple focal nodular hyperplasia of the liver associated with vascular malformations of various organs and neoplasia of the brain: a new syndrome. Mod Pathol 1989;2(5):456-62.

20. Dokmak S, Paradis V, Vilgrain V, et al. A single-center surgical experience of 122 patients with single and multiple hepatocellular adenomas. Gastroenterology 2009;137(5):1698-705.

21. Rebouissou S, Amessou M, Couchy G, et al. Frequent in-frame somatic deletions activate gp130 in inflammatory hepatocellular tumours. Nature 2009;457(7226):200-4.

22. Bromberg J, Wang TC. Inflammation and cancer: IL-6 and STAT3 complete the link. Cancer Cell 2009;15(2):79-80.

23. Bergquist A, Ekbom A, Olsson R, et al. Hepatic and extrahepatic malignancies in primary sclerosing cholangitis. J Hepatol 2002;36(3):321-7.

24. Gow PJ, Chapman RW. Simultaneous occurrence of focal nodular hyperplasia and primary sclerosing cholangitis in a young female. Eur J Gastroenterol Hepatol 2000;12(5):565-7.

25. Garioud A, Seksik P, Chretien Y, et al. Characteristics and clinical course of primary sclerosing cholangitis in France: a prospective cohort study. Eur J Gastroenterol Hepatol 2010;22(7):842-7.

26. Isomoto $\mathrm{H}$. Epigenetic alterations in cholangiocarcinoma-sustained IL-6/STAT3 signaling in cholangio- carcinoma due to SOCS3 epigenetic silencing. Digestion 2009;79 Suppl 1:2-8.

27. Wehbe H, Henson R, Meng F, et al. Interleukin-6 contributes to growth in cholangiocarcinoma cells by aberrant promoter methylation and gene expression. Cancer Res 2006;66(21):10517-24.

28. Aoki CA, Dawson K, Kenny TP, et al. Gene expression by PBMC in primary sclerosing cholangitis: evidence for dysregulation of immune mediated genes. Clin Dev Immunol 2006;13(24):265-71.

29. Kobayashi S, Werneburg NW, Bronk SF, et al. Interleukin-6 contributes to Mcl-1 up-regulation and TRAIL resistance via an Akt-signaling pathway in cholangiocarcinoma cells. Gastroenterology 2005;128(7):2054-65. 
Hsu et al. - 10 -

\section{FIGURE and LEGENEDS}
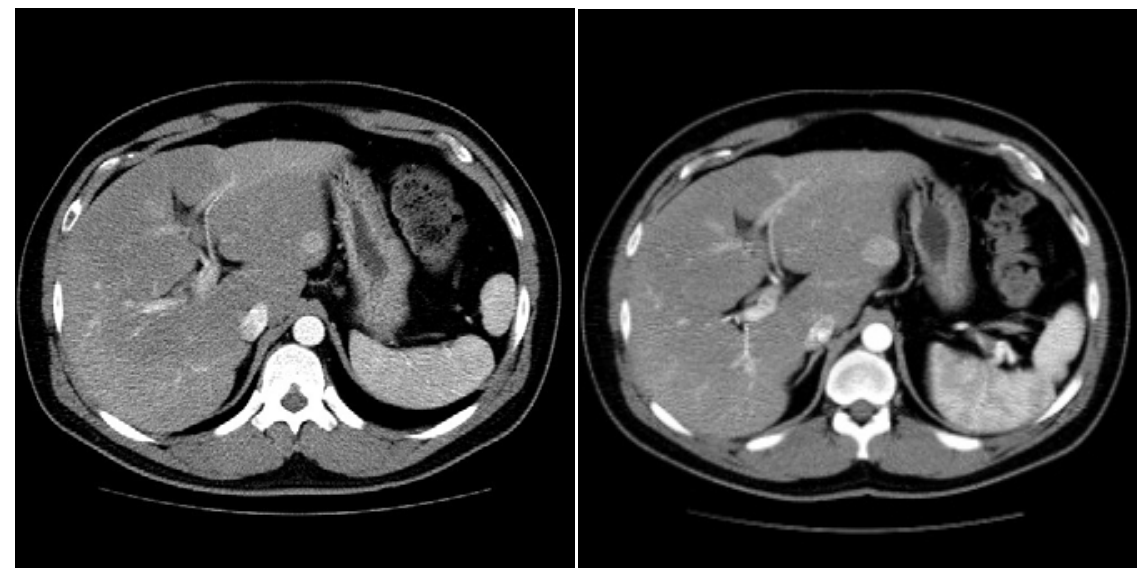

A

B

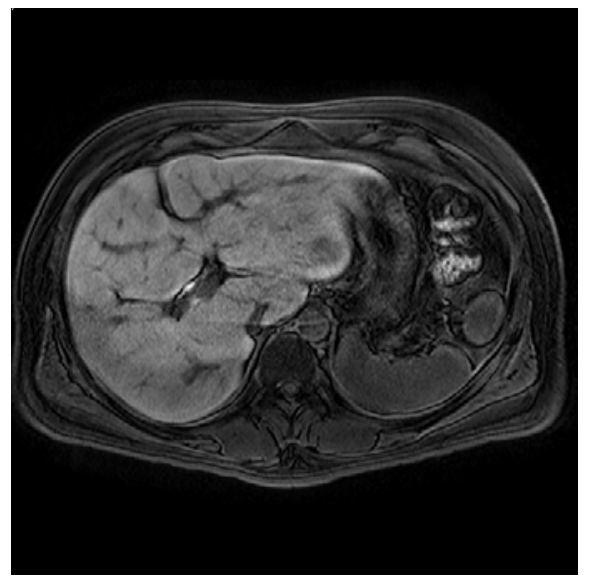

$\mathrm{C}$

Fig.1 Dynamic contrast CT scan (initial CT; A, follow-up CT; B) and Gd-EOB-MRI (C). On a dynamic contrast CT scan, the mass (arrows) revealed enhancement in the hepatic arterial phase. This mass became enlarged from $2.0 \mathrm{~cm}$ (A, in April 2008) to $2.8 \mathrm{~cm}$ (B, in February 2009). No other masses were found in the liver. On the hepatobiliary phase of the Gd-EOB-MRI, the mass showed hypo-intensity (C, arrow). 


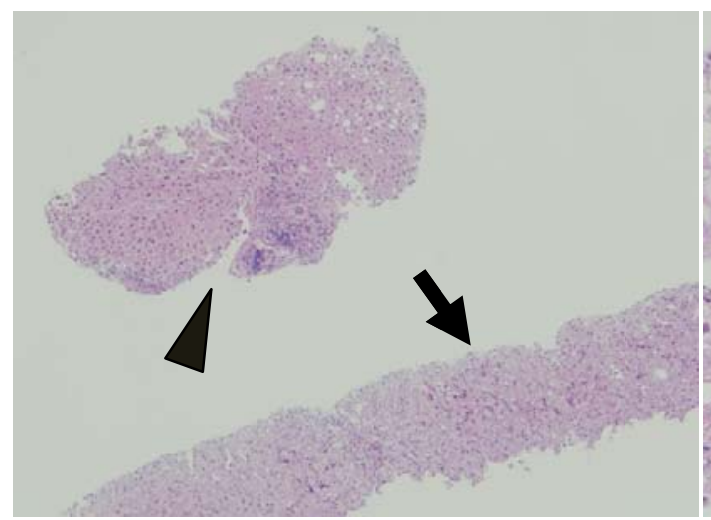

A

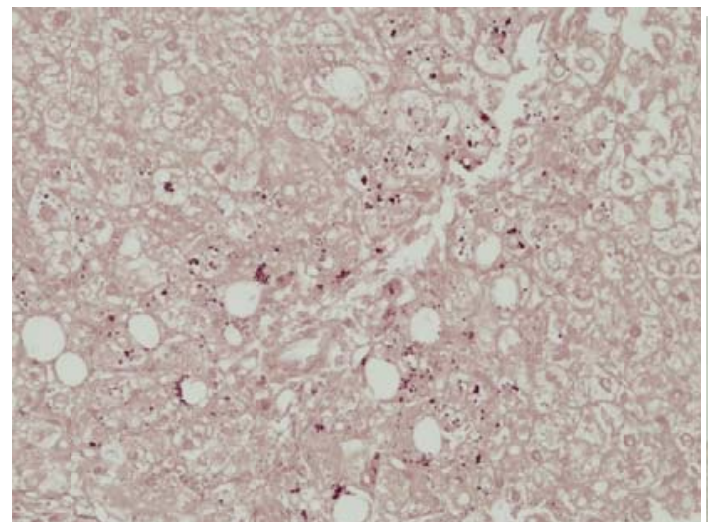

C

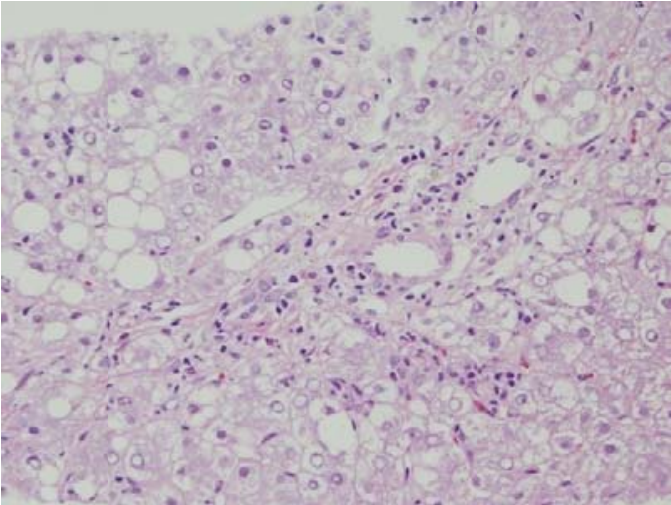

B

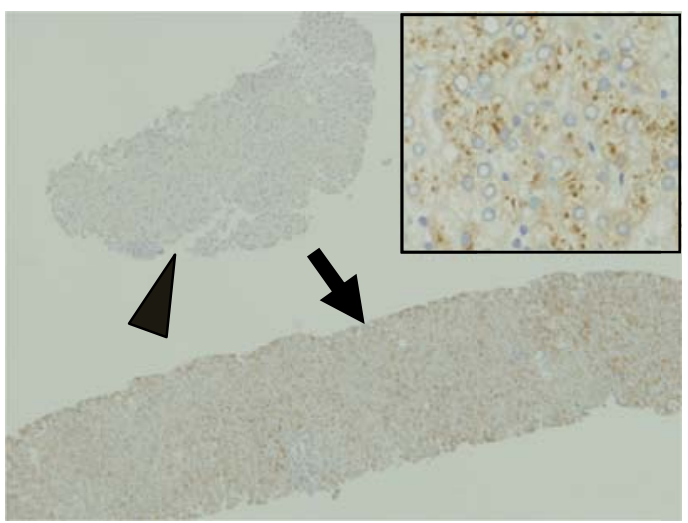

D

Fig.2 Liver needle biopsy. A: Specimens contained tumorous (arrow) and background areas (arrowhead). B: Tumorous lesion composed of uniform hepatocyte-like cells without cellular atypia and glycogenated nuclei were scattered. Moreover, portal tract-like structures lacking bile ducts and mild lymphocytic infiltration were found in tumorous specimen. C: Orcein staining. Orcein-positive copper-binding proteins were scattered around portal tract-like structures. D: Immunostaining for serum amyloid A. Most tumor cells were positive in granular cytoplasmic staining pattern (arrow), though background liver was negative (arrowhead). Inset is a higher magnification of tumorous lesion. 
Hsu et al. - 12 -
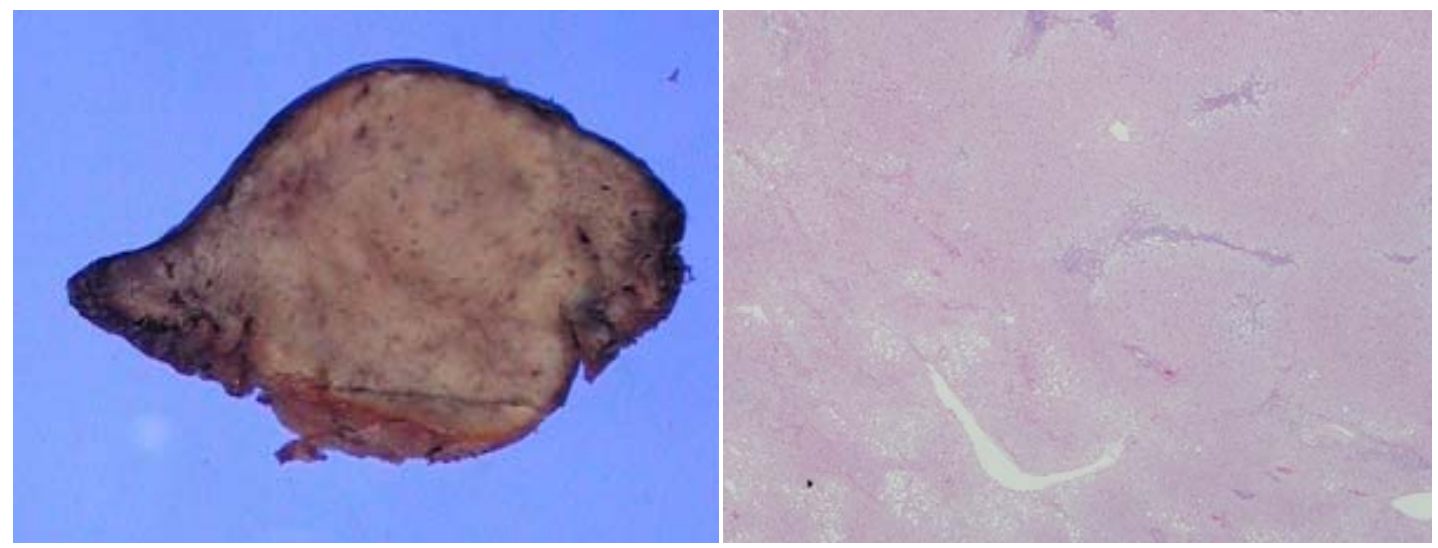

A

B
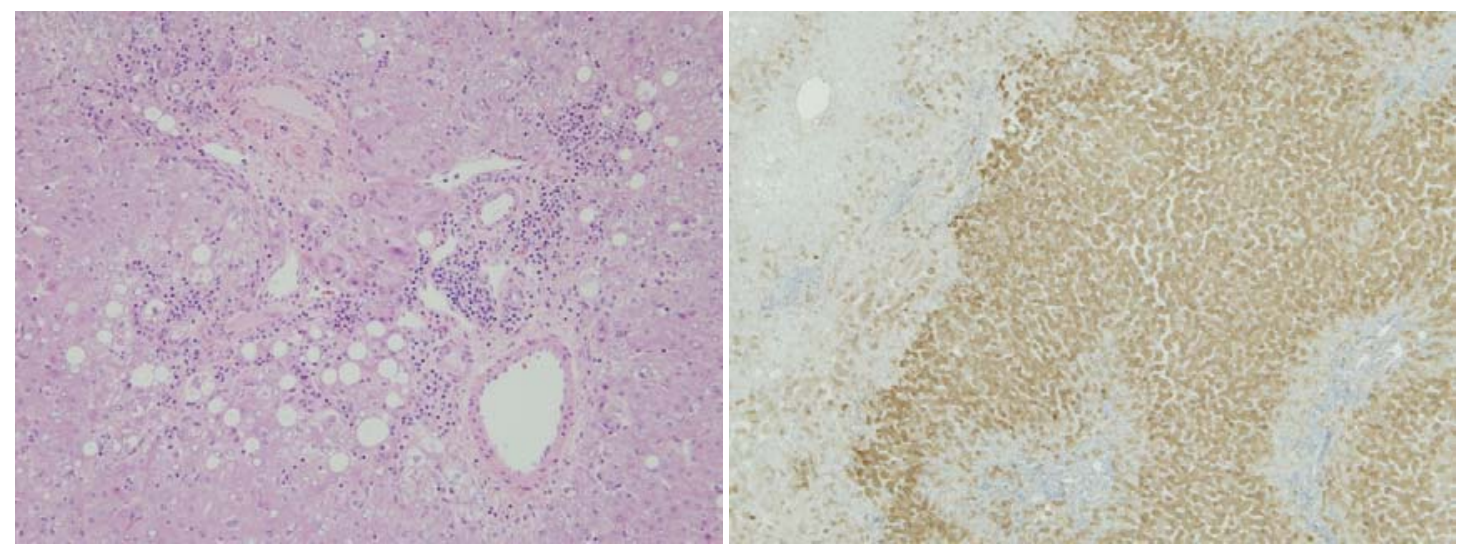

C

D

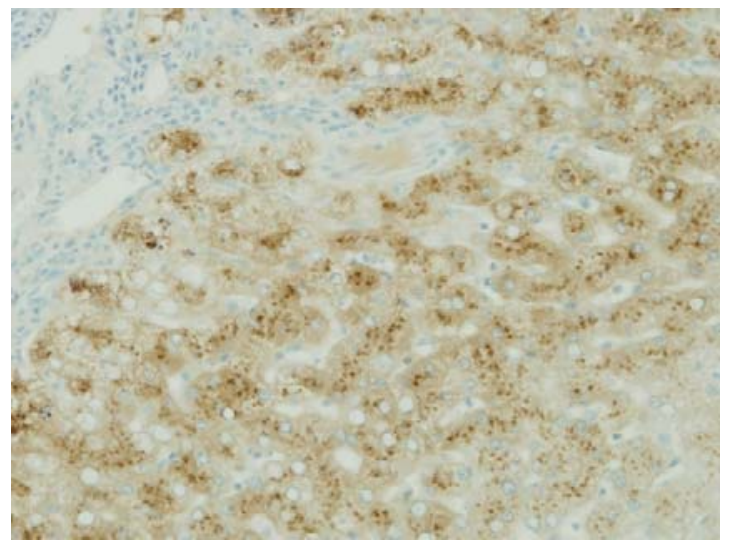

E

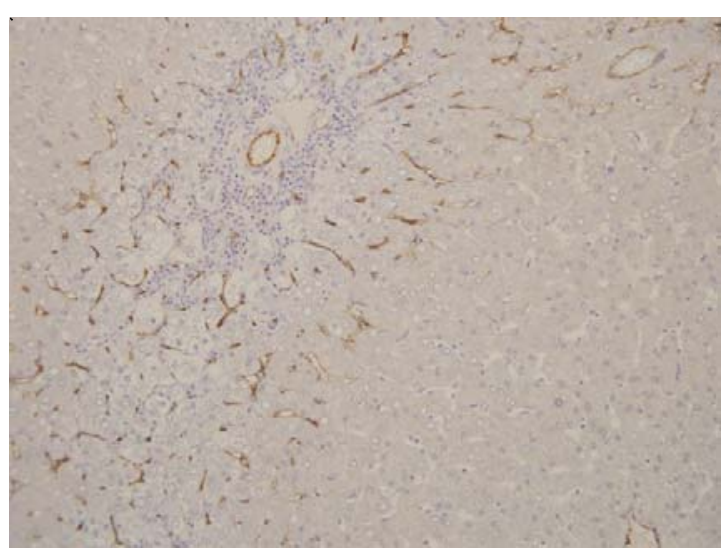

F 


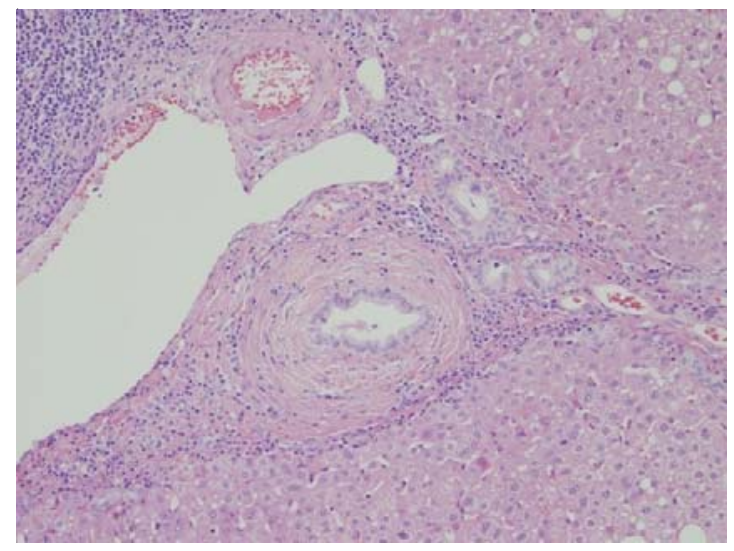

G

Fig.3 Surgically resected liver. A: A non-encapsulated, well-circumscribed $2.6 \mathrm{~cm}$ soft tan nodule was found in the hepatic subcapsular area. Bar indicates $1 \mathrm{~cm}$. B: Nodule was well-demarcated and portal tracts-like structures were found within the nodule. Arrowheads denote a border of nodule. C: Around portal tract-like structures containing several muscular arteries (arrows), brisk lymphoplasmacytic infiltration and macrovesicular steatosis were scattered. D: Immunohistochemistry for C-reactive protein (CRP). Strong positivity was found in nodule. E: Immunohistochemistry for serum amyloid A (SAA). Granular cytoplasmic staining was found. F: Immunohistochemistry for CD34 highlighted the arteries (arrow) within the portal tract-like structures and also showed a sinusoidal inflow staining pattern (arrowheads). G: Background liver. In portal tracts, mild to moderate lymphoplasmacytic infiltration and concentric fibrosis surrounding bile ducts (onion-skin like lesion, arrow) indicating primary sclerosing cholangitis were found. 